\title{
Minireview \\ Control of oncogenesis and cancer therapy resistance
}

\author{
R Perona*,I and I Sánchez-Pérez' \\ 'Instituto de Investigaciones Biomédicas CSIC-UAM, C/Arturo Duperier, 4, Madrid 28029, Spain
}

\begin{abstract}
Despite the combined action of surgery, radiotherapy and chemotherapy, the leading cause of death in cancer patients continues to be the acquired, or intrinsic, tumour resistance to therapy. Some of the genetic alterations that contribute to the malignant transformation are involved in maintaining cell survival under uncontrolled growth conditions. Chemotherapy agents, as well as radiotherapy, trigger a series of signalling pathways in the cells that activate not only the apoptotic machinery, but also cell-survival pathways. In this scenario, the efficacy of therapy is the result of balance between the apoptotic and the survival pathways activated in the tumour, and those elicited by the therapeutic agent. Apoptosis is one of the programmes usually altered in most cancers so as to guarantee tumour progression and, often, these alterations are responsible for therapy resistance, as well.
\end{abstract} British Journal of Cancer (2004) 90,573-577. doi:I0.1038/sj.bjc.660I552 www.bjcancer.com (C) 2004 Cancer Research UK

Keywords: oncogenes; drug resistance; radiotherapy; apoptosis; chemotherapy

\section{ONCOGENIC TRANSFORMATION AND SURVIVAL PATHWAYS}

Therapy resistance mechanisms that tumour cells develop are complex and can be acquired during, or after, treatment. This aggressive behaviour is often derived from survival pathways activated during malignancy progression and driven by oncogenic transformation. There are several examples of oncogenes that can activate such survival pathways, and among these are ras, src, raf, kit, together with the amplification of HER2 and EGFr. The paradigm of these oncogenes is ras, because of the involvement of mutations in ras itself in about $20 \%$ of human tumours. In other cases, EGFr overexpression or HER2-neu amplification positively regulate ras signalling pathways (Downward, 2003). Both, EGFr and Her2 expression are altered in many types of cancer, including those of the breast, ovary and stomach. Additionally, truncation of the EGFr, which results in deletion of the extracellular domain, is found in a significant proportion of glioblastomas and other tumour types. The EGFr family of tyrosine kinases is also activated by the autocrine production of EGF-like factors, such as TGF- $\alpha$, in tumours. In addition to the raf/MAPK pathway involved in cell cycle activation, ras directly activates the catalytic subunit of the type I phosphatidylinositol-3 kinase (PI3K) that leads to the production of phosphatidylinositol 3,4,5 triphosphate, a second messenger that interacts with a large number of downstream enzymes such as AKT/PKB. AKT has a strong antiapoptotic action by phosphorylating several targets, and is the main survival pathway activated by ras. The mechanism by which AKT protects cells from apoptosis is by its ability to phosphorylate several components of the cell-death machinery (Vivanco and Sawyers, 2002). AKT inhibits the catalytic activity of caspases by phosphorylation. Bad, a proapoptotic member of the bcl2 family of proteins, is also phosphorylated by AKT, thus preventing its

* Correspondence: R Perona; E-mail: RPerona@iib.uam.es Received 4 August 2003; revised 4 November 2003; accepted II November 2003 interaction with bclxl and abolishing its proapoptotic function. AKT also phosphorylates FKHR, a member of the forkhead family of transcription factors, thus preventing its nuclear translocation and inhibiting expression of its targets that include several proapoptotic proteins such as FasL and Bim. Finally, AKT phosphorylates p65, a subunit of the NFKB transcription factor, thus enhancing its transactivation potential and facilitating the expression of several antiapoptotic genes such as BCLxl, and the cIAP family of caspase inhibitors.

Different components of the PI3K pathway are also mutated in several types of tumours. The gene encoding p105, the catalytic subunit of PI3K, is found to be amplified in some ovarian cancers, while AKT has been observed to be amplified in ovarian and breast cancer (Ruggeri et al, 1998). PTEN, a 3-lipid phosphatase that converts PIP3 back to PIP2, acting as a suppressor gene, is deleted or mutated in several human cancers including those of the breast and endometrium, hepatocellular carcinomas, glioblastomas, lung cancer, renal carcinoma and thyroid tumours (Ali et al, 1999).

Several preclinical studies have reported a direct correlation between oncogenic transformation and drug resistance. Examples are the increase in resistance to radiation in bladder carcinomas induced by ras mutations, to CPT-11 and gemcitabine in colon cancer, to gemcitabine in pancreatic carcinoma, and to radiation and BCNU in glioblastomas (Chakravarti et al, 2002). In most of these cancers, resistance to therapy is due to upregulation of PI3K activity. In breast cancer, amplification of HER2 is associated with taxol resistance (Witters et al, 2003), and in glioblastomas increase in EGFr activity to radiation and BCNU (Chakravarti et al, 2002).

Some clinical studies have shown correlations between ras and myc mutations and resistance to therapy in ovarian cancer, and overexpression of EGFr in breast cancer. However, further studies are needed to provide a better overview of the problem, at the clinical level.

c-kit is a type II tyrosine kinase that activates different signalling pathways in response to the ligand: stem cell factor. Mutations in the catalytic activity domain in the cytoplasmic region are associated with transformation in patients with gastrointestinal 
tumours (GIST), a very resistant type of cancer (Hirota et al, 1998). Among the different signalling pathways associated with c-kit activation is the PI3K/Akt survival pathway that contributes to resistance to the different treatments.

p53, a tumour-suppressor gene, plays a central role in DNA damage detection, cell cycle transitions and apoptosis control in response to damage. Since most of the treatment protocols include DNA-damaging agents, the function of p53 is very important in therapeutic response. About $50 \%$ of all human cancers harbour mutations in the p53 gene, which affect its ability to activate expression of proapoptotic genes. Conversely, tumours that retain wild-type p53 frequently have modifications and/or defects in the pathways that facilitate the stabilisation of p53 in response to stress. p53 regulates transcription of genes that regulate both the mitochondrial as well as the receptor-activated apoptotic pathways such as APAf-1, Bax, NOXA, PERP, PTEN, PUMA, FAS, etc (Schuler and Green, 2001). p53-mediated activation of apoptotic target genes is also regulated by cofactors such as JMY, ASPP, and other members of the p53 family, p63 and p73. Both the JMY as well as the ASPP family of proteins have been shown, recently, to interact with $\mathrm{p} 53$ and to enhance the promoters of apoptotic target genes such as bax. IASPP1 is an evolutionary-conserved inhibitor of p53 that works as a transforming protein which co-operates with ras, E1A and E7 to transform cells in vitro. Enforced expression of IASPP confers resistance to radiation and cisplatin-induced apoptosis. IASPP expression is upregulated in human breast carcinomas expressing wt p53 and normal levels of ASPP (Bergamaschi et al, 2003).

At the clinical level, the role of p53 and drug resistance has been extensively evaluated by assessing clinical status in relation to response to chemotherapy and overall survival. Although in some studies correlations have been found between tp53 mutations and chemoresistance, such as in lung and ovarian carcinomas, in other types of tumours, the correlation has not been as clear and is due, possibly, to the involvement of other genetic changes that have accumulated in these tumours.

\section{SIGNAL TRANSDUCTION PATHWAYS ACTIVATED BY THERAPY: APOPTOSIS VS SURVIVAL}

Apart from surgery, cancer treatment is based, mainly, on chemotherapy and radiotherapy, and both are cytotoxic agents that induce apoptosis in target cells. Since most cancers are not sensitive to these treatments, the resistance that is developed becomes a major problem at the clinical level. Further, when tumours relapse, the response to treatment is lower than that of the initial tumour. Most antitumour drugs, and also $\gamma$-radiation, are DNA-damaging agents. Since cells are routinely exposed on a daily basis to many such damaging agents, the principal targets of which are proteins and DNA, they develop different mechanisms in an attempt to minimise, or to resolve, any damage before the mitotic process commences. This is so as to pre-empt deleterious mutations being inherited in the daughter cells. Exposure of cells to therapeutic genotoxic agents initiates a series of events leading to activation of a wide range of signalling pathways that regulate cell cycle repair as well as apoptosis, and involves a wide group of genes with diverse functions. Current knowledge of cancer indicates that it is a complicated disease in which, depending on the type of tumour, many pathways are deregulated. Chemotherapy and radiation activate signalling pathways which can be rapidly induced, as well, in response to mitogenic stimulation and which, sometimes, are the same as those that are deregulated in the tumour. Consequently, it is important for the clinician to know the mechanisms by which apoptosis can be induced so that the balance of the signalling pathways may be tipped towards apoptosis.

Although the primary intracellular targets - of action of chemotherapy agents are different, it has become evident that induced cytotoxicity converges, ultimately, on a common pathway that induces apoptosis. Most of these pathways involve the activation of protein kinases. These include the nonreceptor tyrosine kinase c-abl, Lyn, the mitogen-activated protein kinase (MAPK) family, the transcription factor NFkB and the checkpoint proteins. In evolutionary terms, the MAPK pathway is highly conserved, which includes the ERK, JNK and p38 family. These are organised in a module of three kinases that are activated by a phosphorylation cascade. Each MAP kinase subfamily is activated by a specific upstream MAP kinase kinase (MKKs), which phosphorylates the threonine as well as the tyrosine residues within a conserved $\mathrm{T}-\mathrm{X}-\mathrm{Y}$ motif. Activation of the MAP kinase family members leads to phosphorylation of several cellular effector molecules, including protein kinases such as MAPKAPK1, MAPKAP-K2/3, Mnk1/2, as well as transcription factors such as c-jun, ATF-2, MEF2c and CHOP, all of which influence the fate of the cell.

The MAPKs are conserved proteins that regulate the growth, division and death of the cell. The MAPK pathway represents a cascade of phosphorylation events including three pivotal kinases, namely Raf, MEK (MAP kinase kinase) and ERK (MAP kinase). Activation of ERK by chemotherapy agents is controversial and, ultimately, depends on the type of tumour. Cisplatin, an antitumour agent that is widely used in the treatment of several kinds of solid tumours has been shown to cause activation of ERK in ovarian carcinoma (Hayakawa et al, 1999), neuroblastoma and HeLa cells, but not in keratinocytes. Inhibition or ERK activation results in increased sensitivity to cisplatin and taxol-induced cell death in ovarian cancer cell lines and some melanoma cell lines (Mandic et al, 2001), but can result in an increased resistance to cisplatin in HeLa cells.

Activation of the Jun-N-terminal kinase (JNK) pathway has been implicated in apoptotic response to DNA damage, cell stress and cytotoxic drugs. It appears that, in normal cells, the activation of JNK is a proapoptotic event and drives the activation of proapoptotic members of Bcl-2 family, causing an induction of cytochrome $c$ release from the mitochondria. However, there is less consensus regarding the roles of JNK and of c-Jun in tumour cells. The initiation of mitochondrial apoptosis pathways by JNK is independent of its transcription effects, for the most part. In certain cell types, c-Jun overexpression is clearly a basis for resistance to DNA-damaging drugs, and resistance reversal has been observed using antisense RNA against c-Jun (Hayakawa et al, 1999). Conversely, in other cell systems, the absence of c-Jun makes cells more resistant to cisplatin treatment (Sanchez-Pérez and Perona, 1999). As such, there is still considerable controversy regarding JNK activation and its role in apoptosis and/or survival. Both mechanisms are not mutually exclusive, since certain parameters, such as the timing of the signal, could be important. Sustained JNK activation is required for apoptotic signalling and is sufficient for apoptosis in response to cisplatin and irradiation. In contrast, TNF- $\alpha$ causes transient JNK activation and apoptosis is not induced in some cell lines (Liu et al, 1996). These considerations indicate that transient JNK activation (or elevated basal JNK activity) may be important for mediating a survival response to stress, and that chronic JNK activation may contribute to an apoptotic response. A second aspect to consider is the crosstalk between different pathways. For example, the activation of AKT can suppress the apoptotic effects of activated JNK (Kim et al, 2002) or, alternatively, JNK may modulate other signalling pathways that mediate cell survival, such as NF- $\kappa B$ (Sanchez-Pérez et al, 2002). Target genes that have AP1 and NF- $\kappa B-$ responsive elements in their promoters could be differentially expressed according to the relative activity of the different pathways. Recently, Lamb et al (2003) reported that JNK activation, in response to TNF- $\alpha$, increases together with c-jun and JunD levels. As a consequence, JunD induces c-IAP-2 transcription, while, at 
the same time, modulating apoptosis and cell survival in response to JNK activation.

NF- $\kappa \mathrm{B}$ comprises a family of inducible transcription factors that act as regulators of the host immune and inflammatory responses. Also, they have been involved in protecting cells from apoptosis induced by chemotherapy agents, or cytokine treatment. NFKB is a heterodimer composed, among others, of p50 and p65/RelA subunits (Karin et al, 2002). In nonstimulated cells, NF- $\kappa \mathrm{B}$ is found, mainly, in the cytoplasm, in association with a family of inhibitory molecules known as the IKBs. The activation mechanism of NF- $\mathrm{BB}$ involves phosphorylation of the IKBs via the IKB kinase (IKK) signalosome complex. Two different kinases that phosphorylate the IKKs have been described: NFKB-induced kinase and MEKK1. Once the IKBs have been phosphorylated, they are targeted for ubiquitin binding and subsequent degradation by the 26-S proteosome. Free p50/p65 heterodimers translocate to the nucleus, where they activate transcription of NFKB-responsive genes. Most of these genes such as BCLxl, XIAP, c-IAP-1,2 and FasL (Orlowski and Baldwin, 2002) are involved in apoptosis induction. Chemotherapy agents such as doxorubicin, cisplatin and taxol, among others, induce survival signals, depending on NFkB transcription and on the apoptosis pathways, driven mainly by JNK and p38.

A protein that is key in transducing the DNA damage signals to apoptosis is p53. Most chemotherapy agents, and $\gamma$-radiation, induce stabilisation and activation of $\mathrm{p} 53$. The transcription activity of $\mathrm{p} 53$ is important for its apoptotic functions, since it induces the expression of proteins directly involved in apoptosis, such as BAX, NOXA, PUMA, CD95, TRAIL-R1 and TRAIL-R2. Since p53 is mutated in nearly $50 \%$ of human tumours, induction of apoptosis by chemotherapy agents in these tumours can be compromised.

\section{STRATEGIES FOR OVERCOMING THERAPY RESISTANCE}

Over the past decade, numerous strategies have been designed to modulate signalling pathways activated by oncogenes in an effort to inhibit cell growth in very specific ways. Since genetic alterations that accumulate in tumours favour survival of cells exposed to different stress conditions, the response to therapy frequently becomes severely compromised. New molecules that influence specific signalling pathways administered in combination with cytotoxic drugs have become the preferred strategies in preclinical and clinical studies in an attempt to overcome intrinsic drug resistance. We shall, now, proceed to describe some of these strategies.

Restoring p53 activity in tumour cells has a therapeutic potential because p53 is lost in many tumours and results in resistance to platinum compounds and radiotherapy. Different approaches have been tried in restoring wtp53 activity. These include gene transfer of wtp53 and chemical restoration of wt activity. Gene transfer has been accomplished by using replication-deficient adenoviral vectors that express wtp53. Different preclinical studies have demonstrated synergistic activity between re-expression of p53 and chemotherapy in the patient's response to therapy (Nielsen and Maneval, 1998). The combination of wtp53 gene transfer and chemotherapy with cisplatin, doxorubicin, 5-FU, methotrexate or etoposide in human xenograft tumour animal models of the lung, breast, head and neck, prostate and ovarian cancer produced significantly greater apoptosis and tumour growth suppression than any of the drugs alone (Lebedeva et al, 2003). These results have encouraged clinical studies in patients with advanced cancer. At the NIH database, there are, currently, 29 clinical trials employing wtp53 gene transfer in different types of cancers. Some of these trials show that NSCLC treated with cisplatin, vinorelbine or a combination of both resulted in stabilisation, and regression, of tumours. With respect to ovarian cancer, the one study that used cisplatin resulted in an increased median survival compared to chemotherapy alone. Although the results are promising, the limitation of this approach is linked to the development of new and improved vectors that are not toxic, nonimmunogenic and which can transfer the gene to a high proportion of cancer cells.

An alternative approach that has been used to restore p53 activity is the use of small molecules that modify the p53 mutant strain back to wildtype. CP-31398, a styrylquinazoline, restores a wild-type DNA-binding conformation of mutant $\mathrm{p} 53$, and is able to suppress tumour growth in vitro and in vivo. CP-31398 induces apoptosis in p53 mutant cancer cells by activating the bax/ mitochodrial/caspase-9 pathway, suggesting that it can also sensitise tumour cells to cytotoxic drug treatment (Luu et al, 2002).

Targeting the growth factor receptors that are involved in the survival of cancer cells has become a potential strategy for apoptosis induction, either as a single treatment or in combination with traditional therapies. One potential therapeutic target is the epidermal growth factor receptor (EGFr) superfamily. Many different strategies that interrupt EGFr-dependent signalling have been investigated. Monoclonal antibodies have been developed that target members of the EGFr family. These include antibodies to EGFr and monoclonal antibodies to HER2. Cetuximab, a human-murine chimeric IgG monoclonal antibody, competitively binds to the extracellular domain of EGFr, thus preventing tyrosine kinase activation and inducing apoptosis (Herbst and Langer, 2002). Preclinical studies have demonstrated that, in cell lines expressing EGFr as well, there is an increase in the cytotoxic activity of chemotherapy and radiation (Robert et al, 2001). Clinical studies in patients with NSCLC and head and neck cancer are in progress using this antibody in combination with chemotherapy. Trastuzumab (Herceptin), a monoclonal antibody against the extracellular domain of HER2, was designed for use in breast cancers in which HER2 amplification occurs more frequently. A phase III clinical trial for patients with metastatic breast cancer with overexpression of HER2 demonstrated that the addition of trastuzumab to chemotherapy was associated with a delayed time to -progression of the disease (Azzoli et al, 2002). HER2 is found amplified in lung adenocarcinomas and large-cell carcinomas as well, and is predictive of poorer outcomes. Clinical trials with HER2-positive patients with advanced NSCLC failed to improve treatment outcome for most of the patients in the trial, although a small percentage, in whom HER2 was found to be positive by fluorescence in situ hybridisation (FISH), responded to treatment.

Tyrosine kinase inhibitors to EGF receptors have been developed that compete with and prevent the binding of adenosine triphosphate to the tyrosine kinase region. In preclinical studies, these agents cause inhibition of cell proliferation and induce apoptosis in EGFr-positive cell lines. Gefitinib (ZD1839, Iressa), one of the agents currently in the most advanced stage of development, has shown encouraging results in preclinical studies when combined with chemotherapy in NSCLC. The clinical trials in patients with stage III/IV NSCLC using gefinitib, in combination with gemcitabine and cisplatin, failed to demonstrate any improvement in survival rates. Although other clinical studies, with a more strict selection of patients, have been conducted, it is still not clear if the expression of EGFr is necessary for response, or whether its inhibition will represent a clinical benefit of treatment in NSCLC.

Therapeutic targeting of ras has been approached by inhibiting the covalent attachment of the farnesyl-isoprenoid group to the $\mathrm{H}$ ras, K-ras and N-ras proteins, the first steps in the carboxyterminal post-translational modification of these proteins. The farnesyltransferase inhibitors (FTIs) that have been developed to target RAS also inhibit the farnesylation of other proteins and 
some evidence indicates that the effects observed in tumour growth are due to inhibition of the RAS-related protein RHOB (Prendergast, 2001). This issue, however, remains unresolved at present. Farnesyltransferase inhibitors have shown important effects in growth and survival of some tumour cell lines in vitro and in xenografts in nude mice. Although the results of preclinical studies have been promising, clinical studies have faced several stumbling blocks in their efforts to demonstrate their usefulness in common cancers. Nevertheless, some interesting results have emerged from clinical trials in patients with chronic myeloid leukaemia and other haematological malignancies. Also, in preclinical studies, FTI-277 has been demonstrated to inhibit growth and induce apoptosis in drug-resistant myeloma cells as well as in gliomas when combined with radiotherapy. Interestingly, SCH66336 has been shown to enhance chemosensitivity of human and mouse melanoma cells to cisplatin, and to overcome STI571 resistance in BCR-ABL-positive cells. These results suggest that combination therapy may be more effective in patients with STI571 resistance (Hoover et al, 2002).

The survival-signalling pathway, whose components are subjected to more frequent deregulation in cancer, is that of the PI3K/ AKT pathway, and the consequences of this process contribute to malignancy progression as well as to treatment resistance. As such, chemical inhibitors of AKT have a potential use as suppressors of tumour growth. Early generation examples of this type of compound (wortmannin and LY294002 which inhibit the catalytic activity of p110) have been studied extensively in in vitro assays. LY294002 administration in mouse tumour models has been shown to confer antitumour activity and to enhance the cytotoxicity of treatment with taxol and radiotherapy ( $\mathrm{Hu}$ et al, 2002). Interestingly, in human breast cancer cells, inhibition of PI3K by LY294002 blocks the resistance induced by ras transformation (Jin et al, 2003). The use of rapamycin, an immunosuppressant drug already being used in the clinic and which is an inhibitor of the AKT substrate, mTOR kinase, has opened up the possibility of indirectly inhibiting akt survival pathways. Derivatives of rapamycin, such as CCI-779, have been shown to inhibit the tumour growth of several PTEN-negative tumour cells in vitro and to increase the cytotoxic activity of traditional therapies, Neshat et al (2001). In view of the results obtained with wortmannin, LY294002 and rapamycin derivatives, several drug-discovery programs are underway. The search is for small-molecule inhibitors of PI3K and several other kinases in the pathway, including PDK1 and the integrin-linked kinase (ILK), which might be equally important in keeping the AKT pathway activated.

Activation of the NF- $\kappa \mathrm{B}$-dependent transcription itself, or as a consequence of activated ras/PI3K/AKT pathway, occurs in several human tumours. Additionally, some antitumour agents such as paclitaxel and cisplatin, among others, have induced cell survival via this pathway. Adenovirus-mediated inhibition of NF$\kappa \mathrm{B}$ elicited by the IKB super-repressor protein abrogate the chemoresistance of some types of tumours such as androgenindependent prostate cancer cells and glioma-derived cell lines (Orlowski and Baldwin, 2002). Work is in progress in several laboratories to isolate small molecules that inhibit the IKKs, and whose activities in some tumours lead to constitutive NF- $\kappa \mathrm{B}$ activation and in others, such as breast cancer, where IKK $\alpha$ plays an important role in proliferation. Finally, targeting of IAPs resulting in upregulation by means of the NFKB pathway has been approached via the activation of the apoptosis-ligand receptors of the Apo2/TRAIL, most normal cells being resistant to treatment with TRAIL while cancer cell lines are sensitive to Apo2/TRAIL. Chemotherapy and/or radiation could sensitise TRAIL-induced apoptosis in vitro as well as in vivo. More recently, smac/diabloderived peptides, have been shown to sensitise tumour cells to Apo2/TRAIL or anticancer drug-induced apoptosis in gliomas in vivo (Fulda et al, 2002).

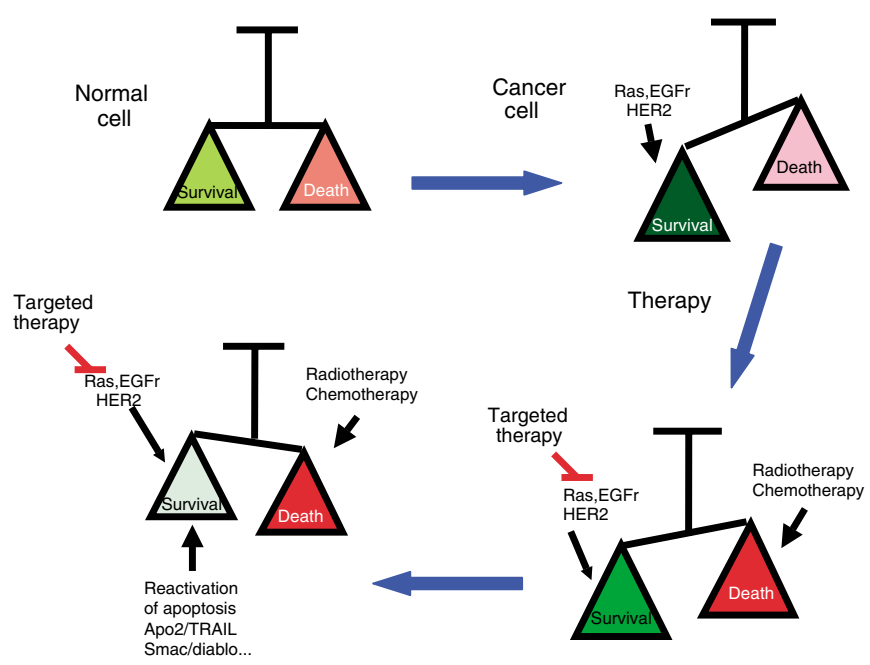

Figure I Combination therapy would shift the balance towards apoptosis in the response of drug-resistant tumours. Note: In normal cells, the balance among apoptosis and survival signals allow controlled homeostasis of the tissue. In cancer cells, survival signals triggered by oncogenic transformation favour uncontrolled growth. Traditional treatments such as chemotherapy and radiotherapy, together with targeted therapies, are still not sufficient to kill tumour cells efficiently due to the existence of antiapoptotic mechanisms in the tumour. Reactivation of the apoptotic machinery by strategies such as Apo2/TRAll delivery would, probably, make the combination of traditional and targeted therapies more efficient.

\section{FUTURE DIRECTIONS}

At the preclinical as well as the clinical level, the problem of drug resistance is still unresolved. Many different approaches have been taken using tumour-derived cell lines and inhibiting signalling pathways activated by oncogenes that participate in cell survival by the administration of combinations of chemotherapy and radiotherapy (Figure 1). One issue that remains unresolved is that of the relative importance of these pathways for tumour survival in vivo, and how they co-operate with genetic changes in the apoptotic machinery in order to ensure tumour progression. Hopefully, more knowledge of the genetic changes that contribute to malignancy in each tumour will be acquired soon, using genomic and proteomic techniques. This would also help in the identification of profiles of expression associated with drug sensitivity or resistance, and this information is used to decide the drug to be used as a first choice. With current knowledge, there are several candidate genes to be taken into consideration, whose activity would be predictive of poor response. Among them are AKT itself and genes that regulate AKT activity, such as PTEN. In this context, several additional aspects need to be taken in consideration. These involve the activity of genes implicated in DNA repair that can condition the efficiency of drug activity in target cells. Last, but not least, therapies that include inhibition of oncogenedependent pathways in combination with traditional therapies and upregulation of apoptosis in tumours by alternative routes (Apo2/ TRAIL, smac/diablo) will, undoubtedly, contribute to the reversal of resistant phenotypes.

\section{ACKNOWLEDGEMENTS}

We would like to thank to Sharmila Chattopadhyay for critically reading this review. We also would like to apologise to all those authors whose work, because of considerations of space, have not been referenced. Work in our group is supported by grants: FIS PI020774 and FIS 01/1094. 


\section{REFERENCES}

Ali IU, Schriml LM, Dean M (1999) Mutational spectra of PTEN/MMAC1 gene: a tumor suppressor with lipid phosphatase activity. J Natl Cancer Inst 91: $1922-1932$

Azzoli CG, Krug LM, Miller VA, Kris MG, Mass R (2002) Trastuzumab in the treatment of non-small cell lung cancer. Semin Oncol 29: 59-65

Bergamaschi D, Samuels Y, O’Neil NJ, Trigiante G, Crook T, Hsieh JK, O'Connor DJ, Zhong S, Campargue I, Tomlinson ML, Kuwabara PE, Lu X (2003) iASPP oncoprotein is a key inhibitor of p53 conserved from worm to human. Nat Genet 33: $162-167$

Chakravarti A, Chakladar A, Delaney MA, Latham DE, Loeffler JS (2002) The epidermal growth factor receptor pathway mediates resistance to sequential administration of radiation and chemotherapy in primary human glioblastoma cells in a RAS-dependent manner. Cancer Res 62: $4307-4315$

Downward J (2003) Targeting RAS signalling pathways in cancer therapy. Nat Rev Cancer 3: $11-22$

Fulda S, Wick W, Weller M, Debatin KM (2002) Smac agonists sensitize for Apo2L/TRAIL or anticancer drug-induced apoptosis and induce regression of malignant glioma in vivo. Nat Med 8: 808-815

Hayakawa J, Ohmichi M, Kurachi H, Ikegami H, Kimura A, Matsuoka T, Jikihara H, Mercola D, Murata Y (1999) Inhibition of extracellular signalregulated protein kinase or c-Jun $\mathrm{N}$-terminal protein kinase cascade, differentially activated by cisplatin, sensitizes human ovarian cancer cell line. J Biol Chem 274: 31648-31654

Herbst RS, Langer CJ (2002) Epidermal growth factor receptors as a target for cancer treatment: the emerging role of IMC-C225 in the treatment of lung and head and neck cancers. Semin Oncol 29: 27-36

Hirota S, Isozaki K, Moriyama Y, Hashimoto K, Nishida T, Ishiguro S, Kawano K, Hanada M, Kurata A, Takeda M, Muhammad Tunio G, Matsuzawa Y, Kanakura Y, Shinomura Y, Kitamura Y (1998) Gain-offunction mutations of c-kit in human gastrointestinal stromal tumors. Science 279: $577-580$

Hoover RR, Mahon FX, Melo JV, Daley GQ (2002) Overcoming STI571 resistance with the farnesyl transferase inhibitor SCH66336. Blood 100: $1068-1071$

$\mathrm{Hu}$ L, Hofmann J, Lu Y, Mills GB, Jaffe RB (2002) Inhibition of phosphatidylinositol $3^{\prime}$-kinase increases efficacy of paclitaxel in in vitro and in vivo ovarian cancer models. Cancer Res 62: 1087-1092

Jin W, Wu L, Liang K, Liu B, Lu Y, Fan Z (2003) Roles of the PI-3K and MEK pathways in Ras-mediated chemoresistance in breast cancer cells. Br J Cancer 89: 185-191

Karin M, Cao Y, Greten FR, Li ZW (2002) NF-kappaB in cancer: from innocent bystander to major culprit. Nat Rev Cancer 2: 301-310

Kim AH, Yano H, Cho H, Meyer D, Monks B, Margolis B, Birnbaum MJ, Chao MV (2002) Aktl regulates a JNK scaffold during excitotoxic apoptosis. Neuron 35: 697-709
Lamb JA, Ventura JJ, Hess P, Flavell RA, Davis RJ (2003) JunD mediates survival signaling by the JNK signal transduction pathway. Mol Cell 11: $1479-1489$

Lebedeva IV, Su ZZ, Sarkar D, Fisher PB (2003) Restoring apoptosis as a strategy for cancer gene therapy: focus on p53 and mda-7. Semin Cancer Biol 13: $169-178$

Liu ZG, Hsu H, Goeddel DV, Karin M (1996) Dissection of TNF receptor 1 effector functions: JNK activation is not linked to apoptosis while NF-kappaB activation prevents cell death. Cell 87: 565-576

Luu Y, Bush J, Cheung Jr KJ, Li G (2002) The p53 stabilizing compound CP-31398 induces apoptosis by activating the intrinsic Bax/mitochondrial/caspase-9 pathway. Exp Cell Res 276: 214-222

Mandic A, Viktorsson K, Heiden T, Hansson J, Shoshan MC (2001) The MEK1 inhibitor PD98059 sensitizes C8161 melanoma cells to cisplatininduced apoptosis. Melanoma Res 11: $11-19$

Neshat MS, Mellinghoff IK, Tran C, Stiles B, Thomas G, Petersen R, Frost P, Gibbons JJ, Wu H, Sawyers CL (2001) Enhanced sensitivity of PTENdeficient tumors to inhibition of FRAP/mTOR. Proc Natl Acad Sci USA 98: $10314-10319$

Nielsen LL, Maneval DC (1998) P53 tumor suppressor gene therapy for cancer. Cancer Gene Ther 5: 52-63

Orlowski RZ, Baldwin Jr AS (2002) NF-kappaB as a therapeutic target in cancer. Trends Mol Med 8: 385-389

Prendergast GC (2001) Actin' up: RhoB in cancer and apoptosis. Nat Rev Cancer 1: $162-168$

Robert F, Ezekiel MP, Spencer SA, Meredith RF, Bonner JA, Khazaeli MB, Saleh MN, Carey D, LoBuglio AF, Wheeler RH, Cooper MR, Waksal HW (2001) Phase I study of anti-epidermal growth factor receptor antibody cetuximab in combination with radiation therapy in patients with advanced head and neck cancer. $J$ Clin Oncol 19: $3234-3243$

Ruggeri BA, Huang L, Wood M, Cheng JQ, Testa JR (1998) Amplification and overexpression of the AKT2 oncogene in a subset of human pancreatic ductal adenocarcinomas. Mol Carcinogen 21: $81-86$

Sanchez-Pérez I, Benitah SA, Martinez-Gomariz M, Lacal JC, Perona R (2002) Cell stress and MEKK1-mediated c-Jun activation modulate NFkappaB activity and cell viability. Mol Biol Cell 13: 2933-2945

Sanchez-Pérez I, Perona R (1999) Lack of c-Jun activity increases survival to cisplatin. FEBS Lett 453: $151-158$

Schuler M, Green DR (2001) Mechanisms of p53-dependent apoptosis. Biochem Soc Trans 29: 684-688

Vivanco I, Sawyers CL (2002) The phosphatidylinositol 3-kinase AKT pathway in human cancer. Nat Rev Cancer 2: 489-501

Witters LM, Santala SM, Engle L, Chinchilli V, Lipton A (2003) Decreased response to paclitaxel versus docetaxel in HER-2/neu transfected human breast cancer cells. Am J Clin Oncol 26: 50-54 